\title{
A New Simple Model for Underwater Wireless Optical Channels in the Presence of Air Bubbles
}

\author{
Emna Zedini ${ }^{1}$, Hassan M. Oubei ${ }^{2}$, Abla Kammoun ${ }^{2}$, Mounir Hamdi ${ }^{1}$, Boon S. Ooi ${ }^{2}$, and Mohamed-Slim Alouini ${ }^{2}$ \\ ${ }^{1}$ College of Science and Engineering, Hamad Bin Khalifa University (HBKU), Doha, Qatar \\ Email: \{ezedini, mhamdi\} @hbku.edu.qa \\ ${ }^{2}$ CEMSE Division, King Abdullah University of Science and Technology (KAUST), Thuwal, Saudi Arabia \\ Email: \{hassan.oubei, abla.kammoun, boon.ooi, slim.alouini\}@kaust.edu.sa
}

\begin{abstract}
A novel statistical model is proposed to characterize turbulence-induced fading in underwater wireless optical channels in the presence of air bubbles for fresh and salty waters, based on experimental data. In this model, the channel irradiance fluctuations are characterized by the mixture ExponentialGamma distribution. We use the expectation maximization (EM) algorithm to obtain the maximum likelihood parameter estimation of the new model. Interestingly, the proposed model is shown to provide a perfect fit with the measured data under all the channel conditions for both types of water. The major advantage of the new model is that it has a simple mathematical form making it attractive from a performance analysis point of view. Indeed, the application of the Exponential-Gamma model leads to closed-form and analytically tractable expressions for key system performance metrics such as the outage probability and the average bit-error rate.
\end{abstract}

Index Terms-Underwater wireless optical communication (UWOC), channel modeling, distribution fitting, maximum likelihood estimation, expectation maximization algorithm, mixture models.

\section{INTRODUCTION}

Underwater wireless optical communication (UWOC) systems have recently attracted considerable research attention as an appropriate and efficient transmission solution for a variety of underwater applications including offshore oil field exploration, oceanographic data collection, maritime archaeology, environmental monitoring, disaster prevention, and port security among others [1]. This rapidly growing interest stems from the recent advances in signal processing, digital communication, and low-cost visible light-emitting diodes (LEDs) and laser diodes (LD) that have the lowest attenuation in seawater [2], [3]. UWOC systems, operating in the blue/green portion of the spectrum in the 400-550 $\mathrm{nm}$ wavelength band, promise high data rates, low-latency, high transmission security, and reduced energy consumption, compared with their acoustic counterparts [1], [4], [5].

Despite their significant advantages, the implementation of UWOC systems is subject to some major challenges. The optical beam can be significantly attenuated by the absorption and scattering effects introduced by the underwater channel [1]. Moreover, UWOC systems are vulnerable to turbulenceinduced fading that results from rapid changes in the refractive index of the water. These refractive index variations are mainly caused by ocean currents, which lead to sudden fluctuations in the temperature and the salinity of the sea water [6]. As a consequence, the received optical intensity undergoes rapid fluctuations which may degrade the UWOC system performance and affect its reliability.

The identification of an accurate description for the absorption and scattering effects in UWOC channels has been extensively addressed in several recent works [7]-[9]. However, turbulence in underwater optical links is relatively less explored as its characterization is more challenging. Moreover, most studies of UWOC directly applied or modified the existing terrestrial free-space optical (FSO) channel models, such as the Lognormal distribution to describe the irradiance fluctuations in the underwater environment [10]-[12]. However, the spectrum of refractive-index variations caused by temperature or pressure inhomogeneities in the atmosphere is much different from the refractive-index spectrum of temperature or salinity in water. This makes the Lognormal distribution not appropriate for modeling the irradiance fluctuations in turbulent water. Therefore, there is a need for further investigation of new accurate statistical models to better characterize the turbulence-induced fading in UWOC.

In [13], the mixture Exponential-Lognormal model has been proposed to describe the irradiance fluctuations in UWOC channels. However, this model is shown to be only valid for specific values of the scintillation index between 0.1 and 1 only. Moreover, the mathematical form of Lognormalbased distributions is not convenient for analytic calculations. Furthermore, the design and the performance analysis of such systems is much more challenging. Indeed, the application of the Exponential-Lognormal in UWOC channels makes it very hard to obtain closed-form and easy-to-use expressions for important performance metrics such as the outage probability and the average bit-error rate (BER). The mathematical intractability of the Lognormal-based model becomes more evident when we know that the assessment of BER is based on numerical methods, as closed-form analytical expressions are not available for this model.

In this paper, we introduce a new simple UWOC turbulence model, that can efficiently characterize irradiance fluctuations from weak to strong turbulence conditions using both fresh as well as salty waters. Based on measured data, we propose the mixture Exponential-Gamma model. We use the expectation maximization (EM) algorithm to obtain the maximum likelihood (ML) estimates of the new model parameters. As we will see in the sequel, the new model not only gives excellent 
agreement with the real measured data under all channel conditions but also serves as a more tractable model when it comes to evaluation of the system performance metrics such as the average BER.

The remainder of this paper is organized as follows. Section II illustrates the experimental setup for intensity fluctuations measurements. In Section III, we introduce the mixture Exponential-Gamma model and we provide its statistical parameters. The EM algorithm is also presented in detail. A comparison between our proposed model and the ExponentialLognormal model is also established by means of statistical goodness of fit tests, presented in Section IV. Section V is devoted to the experimental results and discussion. Finally, some concluding remarks are given in Section VI.

\section{EXPERIMENTAL SETUP}

Fig. 1 illustrates the experimental setup used to collect the data and measure the intensity fluctuations for underwater wireless optical channels under the combined effect of salinity as well as air bubbles induced turbulences. The transmitter is a low cost $520 \mathrm{~nm}$ green LD with a peak emission wavelength of around $515 \mathrm{~nm}$ when biased at $70 \mathrm{~mA}$ and a spectral width (full-width at half-maximum) of $0.45 \mathrm{~nm}$. A plano-convex lens (Thorlabs LA1951-A) of $25.4 \mathrm{~mm}$ focal length is used to collimate and produce a parallel beam. The transmitted power is $7.5 \mathrm{~mW}(8.8 \mathrm{dBm})$. The underwater environment was simulated using $1 \mathrm{~m} \times 0.6 \mathrm{~m} \times 0.6$ water tank made of PVC with $6 \mathrm{~cm} \times 6 \mathrm{~cm}$ acrylic glass windows. The inside of the tank was painted black in order to minimize light reflecting off the sidewalls. Both fresh and salty waters are considered in our measurements. For salinity, we added $118 \mathrm{~g}$ of table salt into the fresh water tank. Air bubbles were generated by a 3/4" PVC pipe with $2 \mathrm{~mm}$ holes placed along the tank. The hole spacing is $5 \mathrm{~cm}$. Five levels of air bubbles were generated, namely $\mathrm{BL}=0 \mathrm{~L} / \mathrm{min}, 2.4 \mathrm{~L} / \mathrm{min}, 4.7 \mathrm{~L} / \mathrm{min}, 7.1 \mathrm{~L} / \mathrm{min}$, and 16.5 L/min. There was no attempt to control the temperature of the water. Using a thermometer, a temperature variation of less than $0.1^{\circ} \mathrm{C}$ was measured throughout the tank.

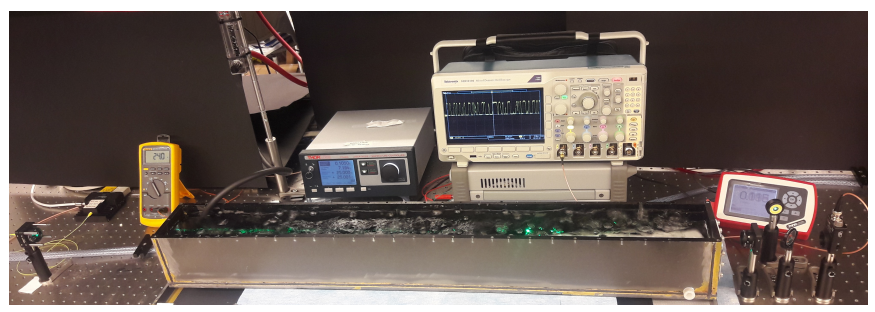

Fig. 1: Actual Photograph of the experimental setup for intensity fluctuations measurements in $1 \mathrm{~m}$ underwater channel in the presence of air bubbles.

After propagating through the $1 \mathrm{~m}$ underwater channel, the $520 \mathrm{~nm}$ beam was focused into a biased silicon PIN photodiode (PD) receiver utilizing a $75 \mathrm{~mm}$ focal length lens (Thorlabs LA1608-A). The technical specifications of the PD (Thorlabs DET36A) include an active diameter of $13 \mathrm{~mm}^{2}$, a responsivity of around $0.19 \mathrm{~A} / \mathrm{W}$ at $520 \mathrm{~nm}$ and a noise equivalent power (NEP) of $0.016 \mathrm{pW} / \mathrm{Hz}^{\frac{1}{2}}$. The output of the PD was captured by a $1 \mathrm{GHz}$ bandwidth mixed domain oscilloscope (Tektronix, MDO 3104) with a sampling rate of $5 \mathrm{GSa} / \mathrm{s}$ for power fluctuations monitoring and measurements. For channel coherence time measurements, we collected 100000 samples with the sampling rate of $100 \mathrm{kS} / \mathrm{s}$. In the case of intensity fluctuations' distribution data, we also collected 100000 samples with sampling rate of $100 \mathrm{~S} / \mathrm{s}$. For all tests, measurements were taken under normal room illumination conditions.

\section{Modeling Underwater Turbulence With the MiXTURE EXPONENTIAL-GAMMA MODEL}

\section{A. Statistics of the New Model}

Throughout this paper, the irradiance fluctuations of the received optical wave, $I$, is modeled by the mixture Exponential-Gamma distribution, which is a weighted sum of the Exponential and Gamma distributions and can be expressed as

$$
\begin{aligned}
& f_{I}(I, \theta)=w f_{\exp }(I, \lambda)+(1-w) f_{\mathrm{gm}}(I, \alpha, \beta) \\
& =\frac{w}{\lambda} \exp \left(-\frac{I}{\lambda}\right)+(1-w) I^{\alpha-1} \frac{\exp \left(-\frac{I}{\beta}\right)}{\beta^{\alpha} \Gamma(\alpha)} \\
& \lambda>0, \alpha>0, \beta>0, I>0,
\end{aligned}
$$

where $\theta=(w, \lambda, \alpha, \beta)$ is the set of parameters with $w$ is the mixture weight or mixture coefficient of the distributions such that $w \in[0,1], \lambda$ is the parameter of the exponential distribution, and $\alpha$ and $\beta$ represent the shape and scale parameters of the Gamma distribution, respectively.

The $n$th moment of $I$ can be given as

$$
\mathbb{E}\left[I^{n}\right]=w \lambda^{n} n !+(1-w) \frac{\beta^{n} \Gamma(\alpha+n)}{\Gamma(\alpha)},
$$

where $\mathbb{E}$ represents the expected value.

We should emphasize that the distribution in (1) has a simpler mathematical form than the Lognormal-based model given in [13, Eq.(8)] and thus lead to straightforward performance evaluation of UWOC systems, with closed-form and mathematically tractable results.

The scintillation index $\sigma_{I}^{2}$, defined as the normalized variance of the intensity fluctuations can be expressed as

$$
\sigma_{I}^{2} \triangleq \frac{\mathbb{E}\left[I^{2}\right]-\mathbb{E}[I]^{2}}{\mathbb{E}[I]^{2}}
$$

By normalizing the received optical power, we get $\mathbb{E}[I]=1$ and therefore the scintillation index can be derived as

$$
\sigma_{I}^{2}=2 w \lambda^{2}+(1-w) \alpha \beta^{2}(1+\alpha)-1 .
$$

\section{B. ML Parameter Estimation of the New Model}

In this paper, we use the EM algorithm to find maximum likelihood estimates of the mixture Exponential-Gamma model parameters. The EM algorithm is an effective iterative method that starts from some arbitrarily initial values for the model parameters $\theta=(w, \lambda, \alpha, \beta)$ such that $\mathbb{E}[I]=$ $w \lambda+(1-w) \alpha \beta=1$ and then proceeds iteratively to update $\theta$ until convergence. 
In EM framework, the set of independent and identically distributed (iid) irradiance observations $I_{1}, I_{2}, \ldots, I_{N}$ is considered as an incomplete data, with $N$ being the number of measured samples. Let $z_{1}, z_{2}$ be a set of missing observations, where the $k$ th element of $z_{i}, z_{k i}=z_{k}\left(I_{i}\right)$, is equal to 1 or 0 according to wether the $i$ th observation of $I, I_{i}$, did or did not arise from the Exponential distribution for $k=1,2$ and $i=1,2, \ldots, N$.

Each iteration of the EM algorithm involves an expectation step, E-step, and a maximization step, M-step [14].

1) E-step: computes the conditional expectation of the hidden variables $z_{k i}$ given the observed data $I_{1}, I_{2}, \ldots, I_{N}$ by using the Bayes's rule as

$$
\begin{aligned}
& \hat{z}_{1 i}=\mathbb{E}\left[z_{1 i} \mid I_{i}\right]=\frac{w f_{\exp }\left(I_{i}, \lambda\right)}{w f_{\exp }\left(I_{i}, \lambda\right)+(1-w) f_{\mathrm{gm}}\left(I_{i}, \alpha, \beta\right)} . \\
& \hat{z}_{2 i}=\mathbb{E}\left[z_{2 i} \mid I_{i}\right]=\frac{(1-w) f_{\mathrm{gm}}\left(I_{i}, \alpha, \beta\right)}{w f_{\exp }\left(I_{i}, \lambda\right)+(1-w) f_{\mathrm{gm}}\left(I_{i}, \alpha, \beta\right)} .
\end{aligned}
$$

2) M-step: uses (5) and (6) calculated in the E-step to update the model parameters via maximization of the log likelihood with respect to $\theta$ as follows

$$
\theta^{(j+1)}=\underset{\theta}{\operatorname{argmax}} \ln L\left(I \mid \theta^{(j)}\right) .
$$

where $\ln L(I \mid \theta)$ is the $\log$ likelihood function that can be written as

$$
\begin{aligned}
\ln L(I \mid \theta) & =\sum_{i=1}^{N} z_{1 i} \ln \left(\frac{w}{\lambda} \exp \left(-\frac{I_{i}}{\lambda}\right)\right) \\
& +z_{2 i} \ln \left((1-w) I_{i}^{\alpha-1} \frac{\exp \left(-\frac{I_{i}}{\beta}\right)}{\beta^{\alpha} \Gamma(\alpha)}\right) .
\end{aligned}
$$

The estimate of the mixing coefficient $\hat{w}$ can be derived as

$$
\hat{w}=\frac{1}{N} \sum_{i=1}^{N} \hat{z}_{1 i}
$$

The parameter $\lambda$ of the Exponential distribution estimated via the ML method is computed using

$$
\hat{\lambda}=\frac{\sum_{i=1}^{N} \hat{z}_{1 i} I_{i}}{\sum_{i=1}^{N} \hat{z}_{1 i}}
$$

The ML estimates of $\alpha$ and $\beta$ parameters of the Gamma distribution in the $j$ th iteration of the EM algorithm are calculated as

$$
\begin{aligned}
& \hat{\alpha}^{(j+1)}=\hat{\alpha}^{(j)} \\
& -\frac{\ln \left(\hat{\alpha}^{(j)}\right)-\psi\left(\hat{\alpha}^{(j)}\right)-\ln \left(\frac{\sum_{i=1}^{N} \hat{z}_{2 i} I_{i}}{\sum_{i=1}^{N} \hat{z}_{2 i}}\right)+\frac{\sum_{i=1}^{N} \hat{z}_{2 i} \ln \left(I_{i}\right)}{\sum_{i=1}^{N} \hat{z}_{2 i}}}{\frac{1}{\hat{\alpha}^{(j)}}-\psi^{\prime}\left(\hat{\alpha}^{(j)}\right)}
\end{aligned}
$$

and

$$
\hat{\beta}=\frac{\sum_{i=1}^{N} \hat{z}_{2 i} I_{i}}{\hat{\alpha} \sum_{i=1}^{N} \hat{z}_{2 i}},
$$

where $\psi(\cdot)$ and $\psi^{\prime}(\cdot)$ are the psi function and its derivative, respectively [15, Eq.(8.360)].

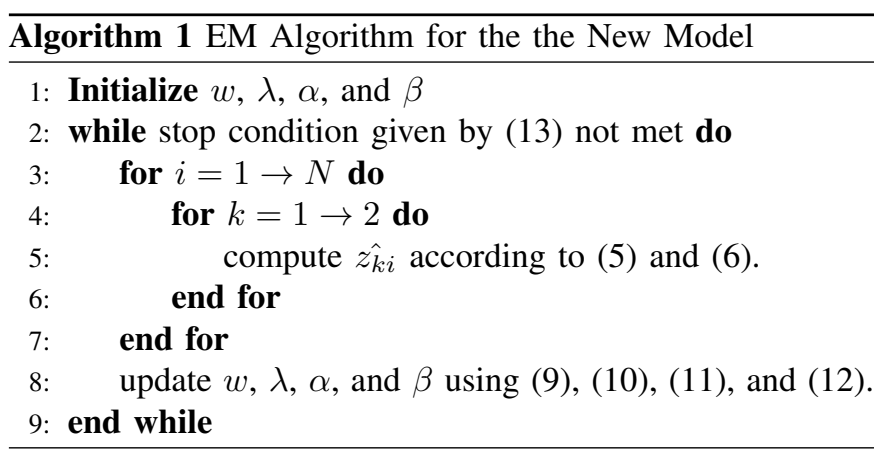

The EM algorithm used to estimate the parameters of the proposed Exponential-Gamma mixture model is illustrated in Algorithm 1.

Convergence of the EM algorithm is reached when the estimate parameters stop changing in a significant manner from one iteration to the next, that is,

$$
\left|\theta^{(j+1)}-\theta^{(j)}\right|<\epsilon
$$

for some $\epsilon>0$.

To compare the new proposed model with the ExponentialLognormal model presented in [13], we have also applied the EM algorithm to obtain ML estimates of the Lognormal distribution parameters, $\mu$ and $\sigma^{2}$, that can be calculated as

$$
\hat{\mu}=\frac{\sum_{i=1}^{N} \hat{z}_{2 i} \ln \left(I_{i}\right)}{\sum_{i=1}^{N} \hat{z}_{2 i}}
$$

and

$$
\hat{\sigma}^{2}=\frac{\sum_{i=1}^{N} \hat{z}_{2 i}\left(\ln \left(I_{i}\right)-\hat{\mu}\right)^{2}}{\sum_{i=1}^{N} \hat{z}_{2 i}} .
$$

The corresponding parameter of the Exponential distribution is estimated and derived using (10).

\section{Goodness of Fit Tests}

The validity of the new proposed model may be verified statistically by conducting goodness of fit tests that describe how well the new model fits to the measured data. Specifically, we use the mean square error (MSE) test and the R-square $\left(\mathrm{R}^{2}\right)$ test that have been widely employed in evaluating the goodness of fit of a variety of fading distributions to channel measurements.

Additionally, by conducting these tests, we compare the Exponential-Gamma distribution with the ExponentialLognormal distribution and we demonstrate that our proposed model can efficiently describe the irradiance fluctuations under all channel conditions for both fresh and salty waters, providing analytical tractability as well. The results of the MSE as well as the $\mathrm{R}^{2}$ tests for both the new ExponentialGamma and the Exponential-Lognormal distributions for different levels of air bubbles under salty and fresh waters are listed in Table I. 


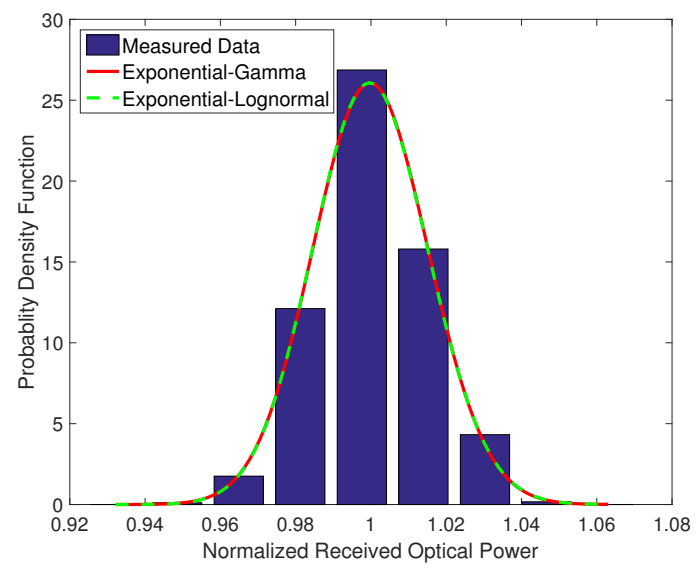

(a) $\mathrm{BL}=0 \mathrm{~L} / \mathrm{min}$.

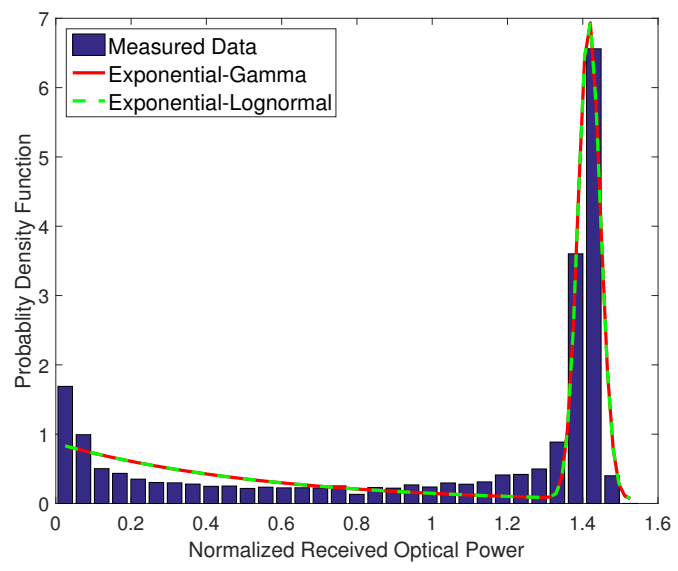

(c) $\mathrm{BL}=7.1 \mathrm{~L} / \mathrm{min}$.

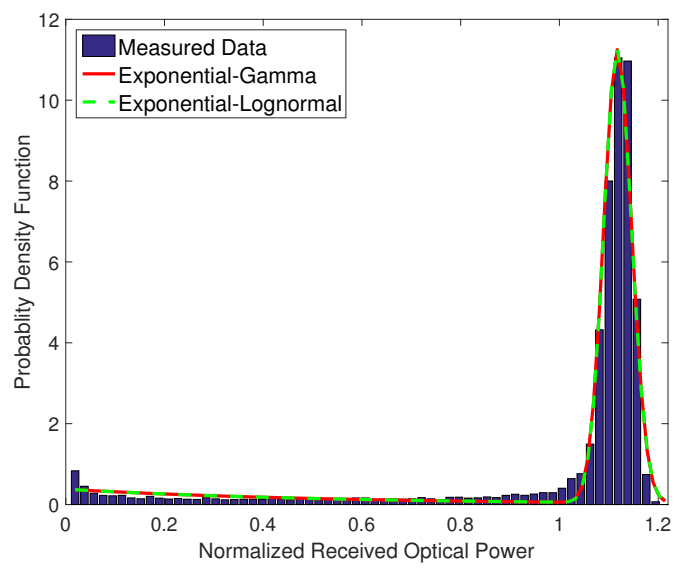

(b) $\mathrm{BL}=2.4 \mathrm{~L} / \mathrm{min}$.

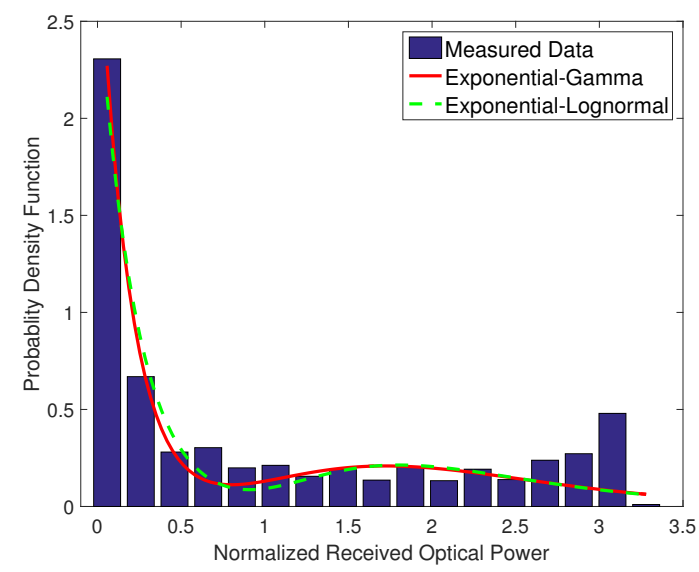

(d) $\mathrm{BL}=16.5 \mathrm{~L} / \mathrm{min}$.

Fig. 2: Histograms of the measured data along with the new Exponential-Gamma as well as the Exponential-Lognormal PDFs for salty water.

\section{A. MSE Test}

The MSE is a simple and efficient measure of how accurately the proposed Exponential-Gamma model predicts the measured irradiance fluctuations. It is defined as

$$
M S E=\frac{\sum_{i=1}^{N}\left[F_{e}\left(I_{i}\right)-F\left(I_{i}\right)\right]^{2}}{N},
$$

where $F_{e}(I)$ denotes the empirical distribution function of $I$ and $F(I)$ stands for the theoretical CDF computed with parameters estimated from the measured data defined as $F(x)=\int_{-\infty}^{x} f_{I}(I, \theta) d I$. It is important to mention here that lower values of MSE (i.e. MSE $\rightarrow 0$ ) indicate a better fit to the acquired experimental data and subsequently a better model.

\section{B. $R^{2}$ Test}

The coefficient of determination, $\mathrm{R}^{2}$, is used to quantify the goodness of fit. $\mathrm{R}^{2}$ is computed from the sum of squared errors, $S S_{\text {err }}$, and the sum of the squares of the distances of the measured points from their mean, $S S_{t o t}$, and can be expressed as [16]

$$
R^{2}=1-\frac{S S_{e r r}}{S S_{t o t}}
$$

where $S S_{\text {err }}=\sum_{i=1}^{M}\left(f_{m, i}-f_{p, i}\right)^{2}$ and $S S_{\text {tot }}=$ $\sum_{i=1}^{M}\left(f_{m, i}-\bar{f}\right)^{2}$, with $f_{m, i}$ and $f_{p, i}$ are the measured and predicted probability values for a given received irradiance level, $M$ represents the number of bins of the data histogram, and $\bar{f}=\sum_{i=1}^{M} \frac{f_{m, i}}{M}$.

It is worth mentioning that the $R^{2}$ measure ranges from 0 to 1 and the higher the value of $R^{2}$ (i.e. $R^{2} \rightarrow 1$ ), the better the proposed model fits the measured intensity through the experiment.

\section{EXPERIMENTAL RESULTS}

In this section, we show how the new proposed Exponential-Gamma model provides an excellent agreement with the measured data under all channel conditions as illustrated in Fig. 2 and Fig. 3 for both salty and fresh waters, respectively. Fig. 2 and Fig. 3 show histograms of the experimental data together with the new proposed PDF as well as the Exponential-Lognormal PDF using different levels of air bubbles, based on the parameters of Table I. Results corresponding to the third and the eighth rows of Table I are not included in Fig. 2 and Fig. 3 due to space limitation.

As shown in Fig. 2 and Fig. 3, our proposed model matches perfectly the measured data for all bubbles levels which 


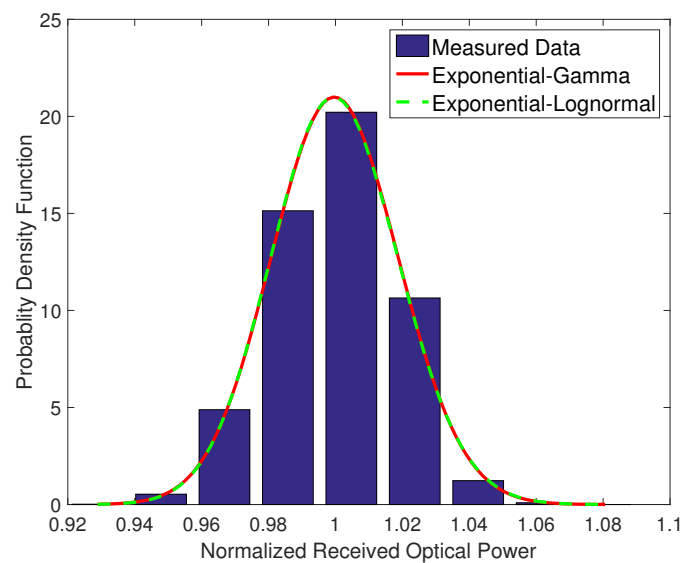

(a) $\mathrm{BL}=0 \mathrm{~L} / \mathrm{min}$.

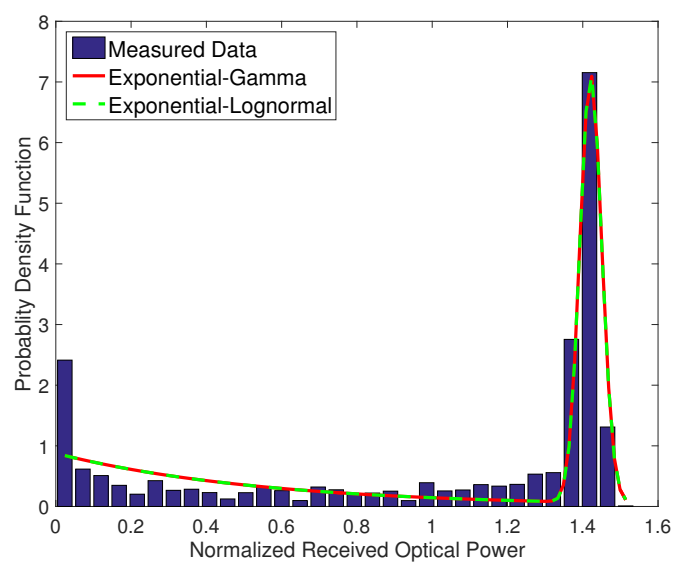

(c) $\mathrm{BL}=7.1 \mathrm{~L} / \mathrm{min}$.

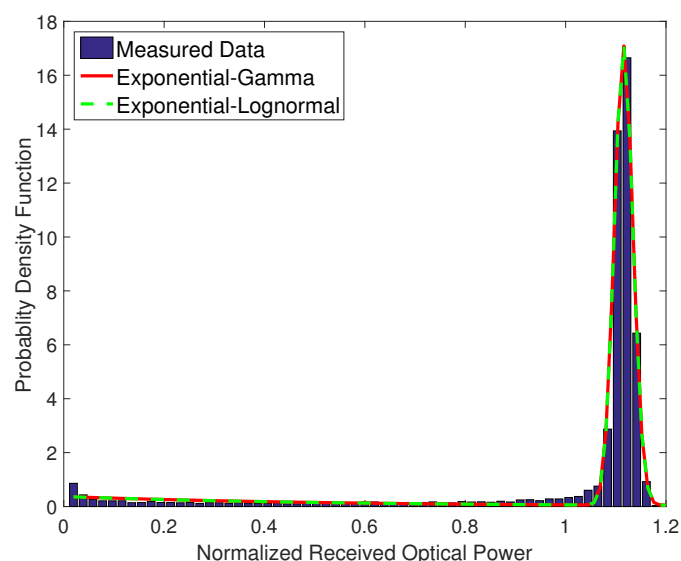

(b) $\mathrm{BL}=2.4 \mathrm{~L} / \mathrm{min}$.

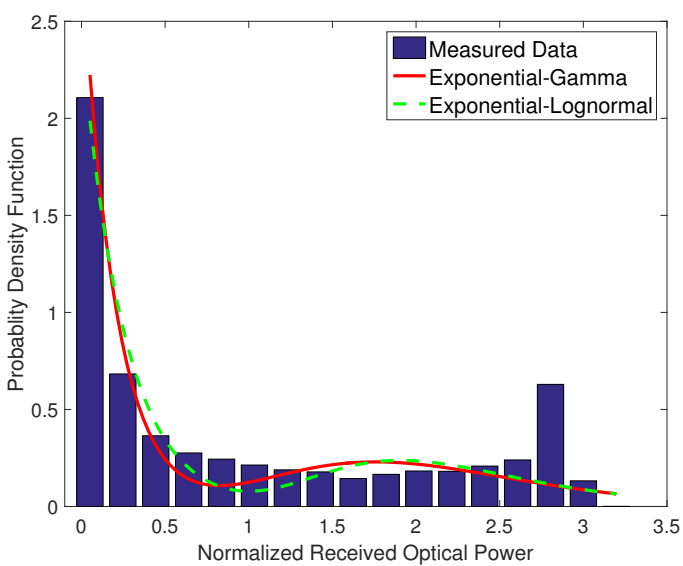

(d) $\mathrm{BL}=16.5 \mathrm{~L} / \mathrm{min}$.

Fig. 3: Histograms of the measured data along with the new Exponential-Gamma as well as the Exponential-Lognormal PDFs for fresh water.

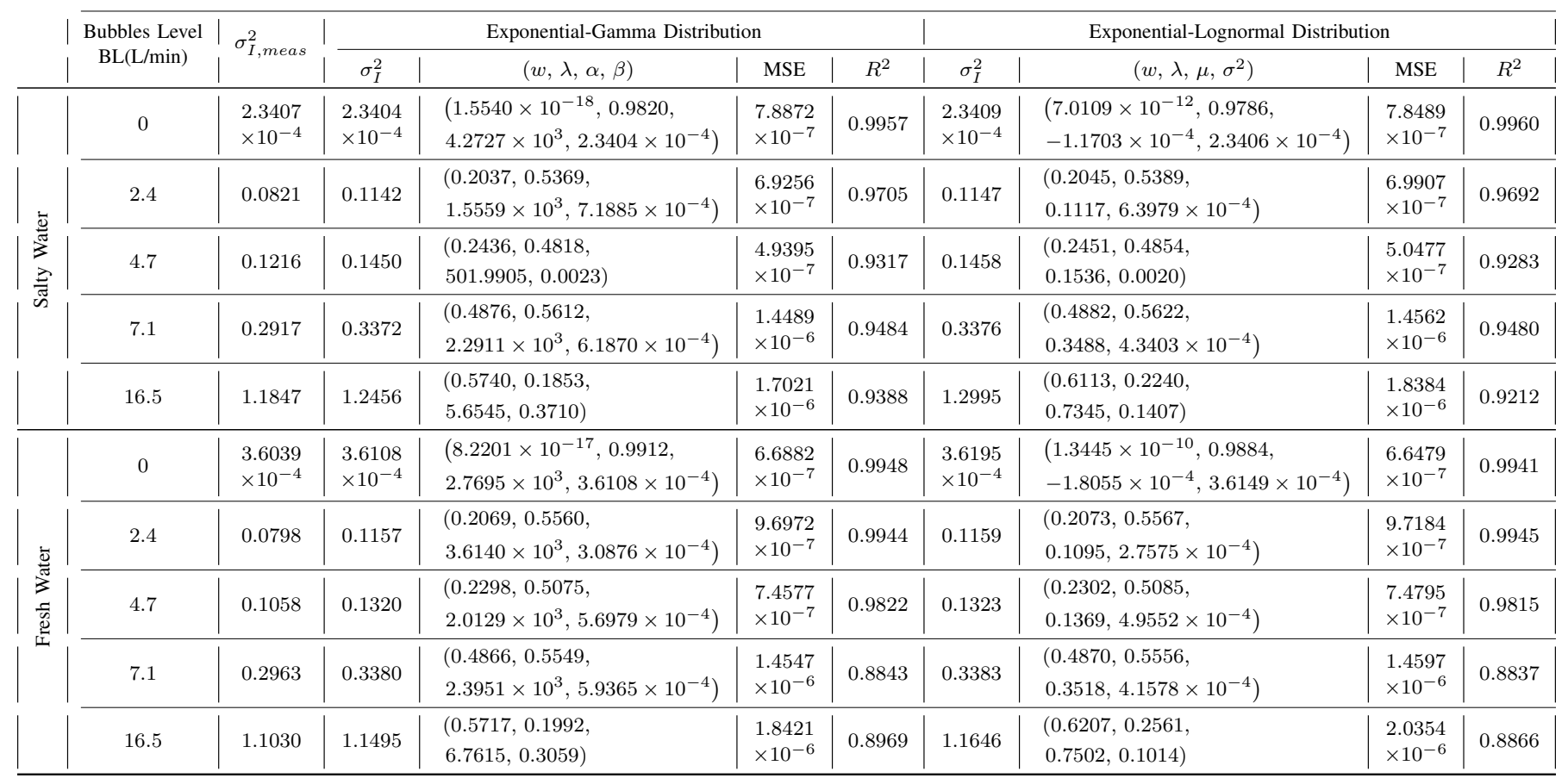

TABLE I: Measured and estimated parameters of the Exponential-Gamma and the Exponential-Lognormal distributions along with the goodness of fits tests. 
correspond to different turbulence conditions varying from weak to strong turbulence, for both fresh and salty waters. This strongly indicates the effectiveness of our model to describe irradiance fluctuations in UWOC channels. Moreover, an excellent agreement between the Exponential-Lognormal model and our proposed model is depicted in Fig. 2 and Fig. 3. Indeed, the plots of the two distributions are almost indistinguishable, and both fit very well to the measured data collected at different levels of air bubbles, for both types of water. These facts make the Exponential-Gamma distribution an attractive model to describe turbulence-induced fading in UWOC channels operating under weak, moderate, and strong turbulence conditions. Interestingly, this new distribution not only provides excellent agreement with the measured data under all conditions of turbulence but also serves as a more tractable model that introduces a lot of analytical facilities in deriving easy-to-use expressions for several performance metrics of UWOC systems such as the outage probability and the average BER.

Table I also compares the scintillation index of the experimental data to the scintillation index of the new model as well as the Exponential-Lognormal model. The scintillation index of the measured data is computed according to (3), and the scintillation indices of the proposed model as well as the Exponential-Lognormal model are calculated theoretically using (4) and [13, Eq.(8)], respectively. As shown in Table I, the scintillation indices calculated from the new PDF and the Exponential-Lognormal PDF are nearly identical, and both are close enough to the scintillation index obtained from the measured data. In addition, we can deduce from Table I that as the level of air bubbles increases, the strength of the turbulence increases, and therefore the scintillation index becomes larger for both fresh and salty waters, as expected.

Furthermore, Table I demonstrates the effect of salinity on turbulence in UWOC channels. Clearly, increasing the salinity of the water by adding $118 \mathrm{~g}$ of table salt into the fresh water tank results in an increase in the scintillation index and therefore the strength of the turbulence becomes stronger.

The results of MSE and $\mathrm{R}^{2}$ goodness of fit tests for both the Exponential-Gamma PDF and the Exponential-Lognormal PDF are listed in Table I. It is clearly illustrated that the MSE values corresponding to the new model are the smallest, under all turbulence conditions for both salty and fresh waters. Furthermore, it can be observed that $\mathrm{R}^{2}$ measures associated with the Exponential-Gamma model have the highest values. These results indicate that the new PDF provides a better fit to the experimental data and therefore strongly support the application of the Exponential-Gamma model for turbulence induced-fading in UWOC channels, as a more accurate and simple alternative to the Exponential-Lognormal model.

\section{CONCLUSION}

In this paper and based on experimental data, we have proposed a new model for UWOC channels, in which the irradiance fluctuations are characterized by the mixture Exponential-Gamma model. We have demonstrated that this model perfectly matches the measured data, collected under different channel conditions ranging from weak to strong turbulence conditions, for both salty as well as fresh waters. A comparison with the Exponential-Lognormal model is also performed, and we have shown that the proposed distribution serves as a universal model which can be applicable under all conditions of irradiance fluctuations, providing a better fit to the experimental data as well. Furthermore, this model being simpler and analytically more tractable, is more convenient for performance analysis and design of UWOC systems. Interestingly, it can be efficiently used to obtain simple and exact closed-form expressions for fundamental system performance metrics such as the outage probability and the average BER.

\section{REFERENCES}

[1] Z. Zeng, S. Fu, H. Zhang, Y. Dong, and J. Cheng, "A survey of underwater optical wireless communications," IEEE Communications Surveys Tutorials, vol. 19, no. 1, pp. 204-238, Firstquarter 2017.

[2] D. Tsonev, H. Chun, S. Rajbhandari, J. J. D. McKendry, S. Videv, E. Gu M. Haji, S. Watson, A. E. Kelly, G. Faulkner, M. D. Dawson, H. Haas, and D. O'Brien, "A 3-Gb/s single-LED OFDM-based wireless VLC link using a Gallium Nitride $\mu$ LED," IEEE Photonics Technology Letters, vol. 26, no. 7, pp. 637-640, Apr. 2014.

[3] Y.-C. Chi, D.-H. Hsieh, C.-T. Tsai, H.-Y. Chen, H.-C. Kuo, and G.R. Lin, "450-nm GaN laser diode enables high-speed visible light communication with 9-Gbps QAM-OFDM," Opt. Express, vol. 23, no. 10, pp. 13 051-13 059, May 2015.

[4] W. Liu, Z. Xu, and L. Yang, "Simo detection schemes for underwater optical wireless communication under turbulence,' Photon. Res., vol. 3, no. 3, pp. 48-53, Jun. 2015.

[5] F. Hanson and S. Radic, "High bandwidth underwater optical communication," Appl. Opt., vol. 47, no. 2, pp. 277-283, Jan. 2008.

[6] R. J. Hill, "Optical propagation in turbulent water," J. Opt. Soc. Am., vol. 68, no. 8, pp. 1067-1072, Aug. 1978.

[7] S. Jaruwatanadilok, "Underwater wireless optical communication channel modeling and performance evaluation using vector radiative transfer theory," IEEE Journal on Selected Areas in Communications, vol. 26, no. 9, pp. 1620-1627, Dec. 2008.

[8] C. Gabriel, M. A. Khalighi, S. Bourennane, P. Leon, and V. Rigaud, "Channel modeling for underwater optical communication," in 2011 IEEE GLOBECOM Workshops (GCW'11), Dec. 2011, pp. 833-837.

[9] — , "Monte-Carlo-Based channel characterization for underwater optical communication systems," IEEE/OSA Journal of Optical Communications and Networking, vol. 5, no. 1, pp. 1-12, Jan 2013.

[10] L. C. Andrews, R. L. Phillips, and C. Y. Hopen, Laser Beam Scintillation with Applications. SPIE Press, 2001.

[11] H. Gerçekcioğlu, "Bit error rate of focused gaussian beams in weak oceanic turbulence," J. Opt. Soc. Am. A, vol. 31, no. 9, pp. 1963-1968, Sep. 2014.

[12] X. Yi, Z. Li, and Z. Liu, "Underwater optical communication performance for laser beam propagation through weak oceanic turbulence," Appl. Opt., vol. 54, no. 6, pp. 1273-1278, Feb. 2015.

[13] M. V. Jamali, P. Khorramshahi, A. Tashakori, A. Chizari, S. Shahsavari, S. AbdollahRamezani, M. Fazelian, S. Bahrani, and J. A. Salehi, "Statistical distribution of intensity fluctuations for underwater wireless optical channels in the presence of air bubbles," in 2016 Iran Workshop on Communication and Information Theory (IWCIT'16), Tehran, Iran, May 2016, pp. 1-6.

[14] T. K. Moon, "The expectation-maximization algorithm," IEEE Signal Processing Magazine, vol. 13, no. 6, pp. 47-60, Nov. 1996.

[15] I. S. Gradshteyn and I. M. Ryzhik, Table of Integrals, Series and Products. New York: Academic Press, 2000.

[16] J. Devore, Probability and Statistics for Engineering and the Sciences, 8th ed. Cengage Learning, 2011. 